\title{
Colza et abeilles : exposé de la problématique et mise en place d'un dispositif de vigilance sur les ruchers en région Centre
}

\author{
Céline ROBERT \\ Cetiom, Avenue Lucien Brétignières, \\ 78850 Thiverval-Grignon \\ $<$ robert@cetiom.fr>
}

\begin{abstract}
The apiarian and agricultural sectors got together to set up an apiary survey in Eure-et-Loir, in 2010, during oilseed rape flowering. The aim of this observatory was to highlight what can occur during oilseed rape flowering regarding bee population evolution and agricultural practices. The year 2010 was marked by important larvae and brood mortalities which induced a national investigation. Among the 4 surveyed apiaries, 2 were also concerned by these mortalities but this could not be explained by the observations and the analysis that were carried out. However, this survey enabled us to identify apiarian and agricultural practices around the apiaries and to combine expert testimonies of apiarian and agricultural professionals.
\end{abstract}

Mots clés : Apis mellifera, oilseed rape, mortalities, apiary survey, apiarists, farmers

Or, depuis une vingtaine d'années, des mortalités d'abeilles domestiques et sauvages anormales sont constatées dans de nombreux pays du monde. Les taux de remplacement des colonies étaient de $10 \%$ en 1980 et sont plutôt, à l'heure actuelle, de l'ordre de $36 \%$. Le maintien d'un cheptel de colonies d'abeilles domestiques productif est devenu aujourd'hui l'une des préoccupations majeures dans les exploitations apicoles. Les causes de mortalité admises par la communauté scientifique internationale sont multiples:

- les parasites ou les pathogènes (Varroa destructor, Nosémoses...);

- la raréfaction des sources de nourriture liée à l'intensification de l'agriculture : diminution des espaces non cultivés et simplification des rotations entraînant une perte de diversité des sources de nourriture ainsi qu'une fragmentation des habitats ;

- les produits phytosanitaires, en particulier les insecticides. Pour être autorisés, ces produits ont fait l'objet d'études spécifiques quant à leur impact sur les abeilles, ce qui a conduit à des préconisations adaptées. Certains produits sont totalement interdits en période de floraison, d'autres peuvent être utilisés, mais toujours hors des périodes de butinage. Une mauvaise utilisation des produits phytosanitaires, par exemple en présence des abeilles dans les parcelles (utilisation interdite par la réglementation actuelle) peut être à l'origine d'accidents.

D'autres éléments peuvent expliquer ces déclins de populations tels que des problèmes génétiques ou des conditions climatiques exceptionnelles.

Les principales causes de mortalités sont d'autant plus difficiles à déterminer qu'elles sont variables selon les régions du monde (Mollier et al., 2009) et que des interactions complexes entre les différents facteurs explicatifs ne sont pas à écarter.

Le Cetiom (Centre technique des oléagineux et du chanvre industriel) travaille principalement sur le colza, le tournesol, le soja, le lin et le chanvre industriel. II $s^{\prime}$ intéresse de près aux problèmes des abeilles, pour plusieurs raisons.

Tout d'abord, le colza est une culture mellifère très importante. De par sa floraison très précoce et également son importance en termes de surface cultivée, elle constitue une des premières sources de nourriture dans de nombreu(GEM-ONIFLHOR, 2005) (figure 1). 


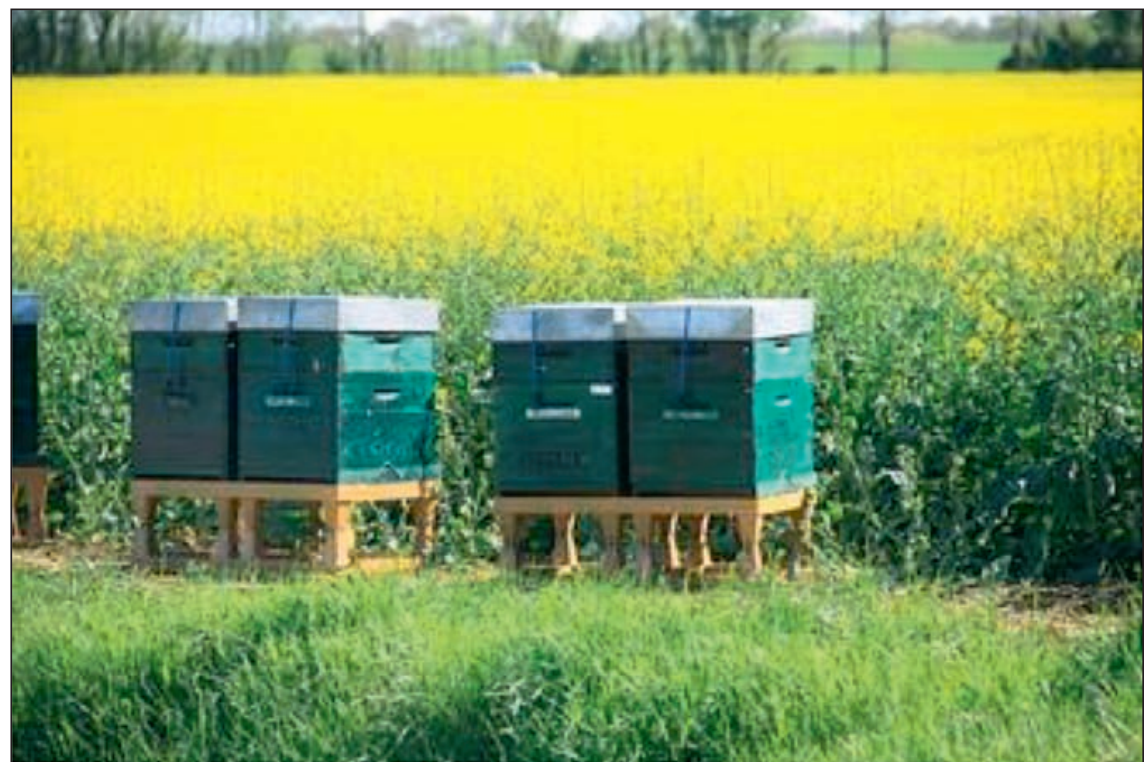

Figure 1. Rucher en bordure d'un champ de colza (photographie de Vincent Ortoli).

ses régions à la sortie de l'hiver. Ainsi, dans les principales zones de production de colza, en moyenne, $60 \%$ de la production de miel se fait sur cette culture. Or, depuis une vingtaine d'années, les apiculteurs observent une diminution du nombre d'abeilles dans les ruches après la floraison du colza mais également une production de miel relativement faible par rapport à ce qui pourrait être attendu.

De plus, il a été montré que les insectes, dont les abeilles, contribuent à la pollinisation du colza pour $30 \%$ pour le colza classique et pour $90 \%$ pour la production de semences de colza hybrides (Pierre et Renard, 2010) où une fécondation croisée est indispensable. Des apiculteurs sont ainsi employés par les producteurs de semences pour placer leurs ruches à proximité des parcelles.

En France, les relations sont souvent difficiles entre le monde apicole et agricole. Les produits phytosanitaires ont été particulièrement montrés du doigt pour expliquer les mortalités anormales, en particulier les insecticides systémiques, c'est-à-dire capable de migrer dans tous les organes de la plante, tels que ceux à base d'imidaclopride, de thiametoxam, de thiaclopride. .

Des études sont donc mises en place par le Cetiom depuis 2010, avec différents partenaires, afin de :

- rétablir le dialogue entre le monde apicole et agricole ;
- développer des méthodologies (existantes ou non) d'observation d'abeilles ou de colonies dans le but de fournir des données les plus fiables possibles pour décrire les phénomènes de mortalité observés en France.

En 2010, le Cetiom, la FDSEA Eure-etLoir, la FRSEA Centre, la section apicole de la FNSEA, 3 apiculteurs et Bayer CropScience se sont associés afin de mettre en place une action pilote en Eure-et-Loir avec l'appui du prestataire de service Staphyt. II s'agissait d'observer dans des conditions habituelles de pratiques apicoles et agricoles le comportement de colonies d'abeilles, homogènes entre elles, pendant la période de butinage du colza. Ces observations ne constituent pas une expérimentation et $\mathrm{n}^{\prime}$ ont donc pas pour vocation première $d^{\prime}$ expliquer les phénomènes de mortalités de colonies observés ces dernières années en France mais aussi dans d'autres pays. L'objectif est plutôt de dresser un état des lieux de ce qui peut se passer au cours d'une miellée de colza, tant du point de vue de l'évolution des populations d'abeilles que des pratiques agricoles. Dans le cas d'incidents de mortalités, les données récoltées pourront peut-être permettre de fournir des éléments de compréhension quant aux facteurs pouvant expliquer ou non ces phénomènes, dans le cas bien particulier des ruchers suivis. Cette action a surtout pour but d'apporter les premiers éléments de méthodologie de suivi de rucher, d'ouvrir la voie à de futurs partenariats et permettre de renouer le dialogue entre le monde apicole et le monde agricole.

\section{Matériel et méthodes}

Les 4 ruchers suivis appartiennent à 3 apiculteurs volontaires et sont situés sur quatre sites éloignés de quelques dizaines de kilomètres (figure 2). Chaque rucher est constitué d'une dizaine de ruches choisies de manière à ce qu'elles soient homogènes entre elles. Une ruche a été écartée au dernier moment du fait de l'absence de reine. Trente-neuf ruches ont donc été suivies pendant toute la période de floraison du colza, c'est-à-dire de fin avril à début juin. Deux ruches par rucher sont équipées de trappes à pollen

\section{Observations}

Un état initial et final de chaque ruche est réalisé. Ces évaluations consistent en une estimation de la force des colonies, à savoir :

- une estimation de la taille des colonies ; - le nombre de cadres de couvain et sa composition en termes de proportion d'œufs, de larves et de pupes ;

- les proportions en pollen et en miel ; - I'observation de la reine ;

- un examen clinique (pression Varroa, loque américaine et européenne, nosémose).

\section{Prélèvements :}

Lors des états initiaux et finaux, des prélèvements d'abeilles vivantes et de pain d'abeilles sont également réalisés : des analyses pathologiques et de résidus sont faites sur abeilles vivantes ainsi que des recherches de néonicotinoïdes dans le pain d'abeille. Les échantillons analysés consistent en un échantillon moyen de l'ensemble des ruches d'un rucher. Cependant, les échantillons récoltés par ruche sont conservés et permettent si nécessaire, la réalisation d'analyses complémentaires individuelles.

En complément des états initiaux et finaux des ruches, un suivi hebdomadaire est fait consistant en :

- un prélèvement d'abeilles vivantes par ruche pour analyses de résidus et analyses pathologiques ;

- une observation de présence ou non d'abeilles mortes "à la porte de la ruche " (observations faites en cas d'inci- 


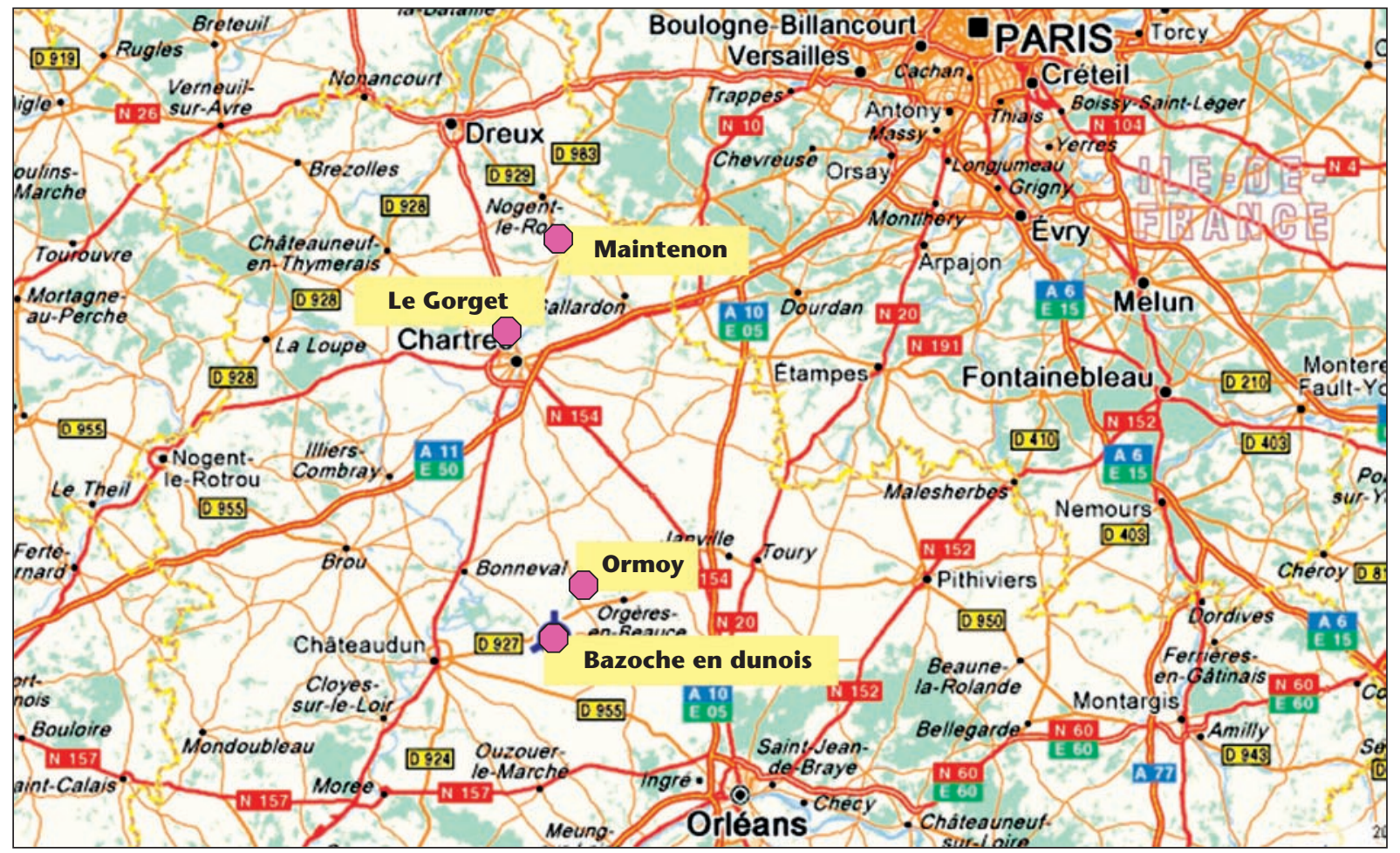

Figure 2. Localisation des 4 ruchers.

dents de mortalité). Dans ce cas, un prélèvement est effectué ;

- un prélèvement de pollen dans les trappes pour analyses palynologiques (composition qualitative et quantitative des origines du pollen) et analyses de résidus (également lors de l'état final).

L'ensemble des prélèvements ainsi que les états finaux et initiaux ont été réalisé en présence des apiculteurs par la société Staphyt.

\section{Analyses}

Le coût des analyses, le matériel nécessaire ainsi que l'impossibilité dans certains cas de disposer d'échantillons individuels en quantité suffisante pour réaliser les analyses ruche par ruche, nous ont conduit à travailler sur des échantillons moyens.

Les analyses multi-résidus demandées ciblent les principales molécules fongicides et insecticides utilisées sur colza, ainsi que les néonicotinoïdes également utilisés sur d'autres cultures comme le thiametoxam (traitement de semences maïs).

Les analyses pathologiques concernent la recherche de nosémose, de varroase et de virus de paralysie chronique.
Les analyses palynologiques sont faites par le Cétam (Centre d'études techniques Apicoles de Moselle), les analyses pathologiques et de recherches de néonicotinoïdes dans le pain d'abeilles par I'ANSES de Sofia-Antipolis (Agence nationale de sécurité sanitaire de I'alimentation, de l'environnement et du travail) et les analyses multi-résidus sur abeilles et pollen par le GIRPA (Groupement interrégional de recherche sur les produits agropharmaceutiques - Angers).

\section{Pratiques agricoles}

En parallèle du suivi des ruches, une enquête auprès des producteurs de colza dans un rayon de $2 \mathrm{~km}$ autour de chaque rucher est menée. Elle vise à inventorier les pratiques phytosanitaires réalisées sur cette culture. Les principaux renseignements demandés sont : les variétés cultivées, les dates de semis, la phénologie, les interventions phytosanitaires (date, cible, produit, dose, volume d'application, conditions climatiques et heures d'intervention). Quelques renseignements concernant d'autres cultures que le colza ont pu être recueillis. Le recueil des observations est centré en priorité sur les interventions entre la reprise de végétation ( $\mathrm{C} 1-\mathrm{C} 2)$ et la fin de la floraison (G5) (tableau 1).

\section{Principaux résultats}

\section{Enquêtes sur les pratiques agricoles}

La très grande majorité des agriculteurs possédant des parcelles dans les 4 zones ont été interrogés. Les pratiques mises en œuvre ne sont pas connues pour, en moyenne, $20 \%$ des parcelles de colza. $\mathrm{Ce}$ manque vient du fait que les agriculteurs n'ont été prévenus que tardivement et ont été difficiles à identifier.

II ressort de ces enquêtes que la réglementation est respectée :

- tous les produits utilisés sont autorisés ; - les doses d'application sont toujours inférieures ou égales aux doses homologuées ;

- la réglementation sur les mélanges est respectée.

Concernant les périodes d'interventions, la plupart des agriculteurs ayant répondu disent appliquer de préférence les insecticides (et même des fongicides) soit tôt le matin, soit tard le soir, 
Tableau 1. Codification de quelques stades repères du colza.

\begin{tabular}{|ll|}
\hline Stade & Description \\
\hline C1 & $\begin{array}{l}\text { Reprise de végétation ; } \\
\text { apparition de jeunes feuilles. }\end{array}$ \\
\hline C2 & $\begin{array}{l}\text { Entre-nœuds visibles; apparition } \\
\text { d'un étranglement vert clair à la } \\
\text { base des nouveaux pétioles. }\end{array}$ \\
\hline G5 & Grains colorés \\
\hline
\end{tabular}

donc en période défavorable à l'activité des abeilles (pour $93 \%$ des traitements pour lesquels cette information est connue). Néanmoins, le nombre d'interventions pour lesquelles cette information n'est pas disponible est de l'ordre de $30 \%$.

\section{Évolution des populations d'abeilles}

Lors de I'état initial, l'état sanitaire des colonies semble bon. Les réserves en pollen et en miel sont bonnes et chaque ruche dispose en moyenne de 5 à 6 cadres de couvain.

Au cours des 3 premières semaines, aucune anomalie n'est observée. Aux alentours du 17-18 mai des mortalités de couvains (larves nécrosées de couleur marron dans des cellules operculées) sont observées sur 2 ruchers. Les mêmes symptômes avaient été observés en Sologne une semaine plus tôt par le propriétaire des ruches. Des cas similaires chez plusieurs apiculteurs de départements différents ont conduit la Brigade vétérinaire à faire des prélèvements et à réaliser une enquête nationale.

Le bilan final révèle donc que sur les ruchers où les mortalités anormales ont été observées, 30 à $40 \%$ des colonies présentent une interruption de ponte et les autres ne sont pas dans un état optimal. Sur les deux autres sites la situation est plus mitigée :

- pour un rucher, $50 \%$ des colonies sont populeuses, les autres sont dans un état moyen à faible ;

- pour le quatrième rucher, 3 colonies sont belles, $20 \%$ sont presque vides mais cela est lié à des problèmes de gestion apicole.

\section{Analyses multirésidus}

Sur les 17 molécules recherchées dans le pollen ou sur abeilles, au moins
11 molécules ont été utilisées sur les zones étudiées (résultats des enquêtes sur les pratiques) entre le 10 avril et fin mai. 5 de ces 11 molécules ont été détectées, voire quantifiées. Au total, sur environ 310 recherches de molécules potentiellement appliquées sur le terrain, des détections ou des quantifications sont faites dans $9 \%$ des cas.

Des recherches de néonicotinoïdes sont réalisées sur pains d'abeilles : des quantifications sont obtenues lors de l'état initial mais jamais lors de l'état final pour 3 ruchers sur 4 . Pour ce dernier rucher qui correspond au rucher se portant le mieux, une quantification apparait lors de l'état final. Les substances actives qui sont détectées ou quantifiées sur les ruchers où des mortalités anormales de couvain ont été observées ne nous ont pas permis d'expliquer les phénomènes de mortalité. Le seul point commun entre ces deux ruchers concerne la quantification de thiamethoxam dans les pains d'abeilles lors de l'état initial et donc sans relation avec les pratiques connues sur le bassin. Afin de vérifier cette hypothèse, des analyses complémentaires et individuelles ciblant les néonicotinoides ont été faites sur les pains d'abeilles initiaux des ruches les plus atteintes. Du thiamethoxam n'est quantifié que sur 2 ruches sur 7. Cette quantification ne permet donc pas d'expliquer les mortalités observées.

Les analyses multi-résidus ne permettent pas de tirer des conclusions directes sur les mortalités qui ont pu survenir sur 2 ruchers sur les 4 .

\section{Analyses pathologiques}

Aucun rucher ne présente de varroase.

Le virus de paralysie chronique est quantifié sur tous les ruchers à, au minimum, une date donnée. Selon I'Anses, les quantifications sont faibles et indiquent que certaines abeilles sont porteuses mais que cela est sans incidence sur la colonie.

2 ruchers sont porteurs de nosémose et de virus de paralysie chronique mais les quantités n'expliquent pas les mortalités observées.

\section{Analyses palynologiques}

Les trappes à pollen n'ont pas toujours bien fonctionnées et pour certaines dates les analyses palynologiques n'ont pu être faites à défaut de quantité, la priorité ayant été donnée aux analyses multirésidus.

Cependant, il apparaît que les sources $d^{\prime}$ alimentation des abeilles sont très diverses : colza, fruitiers, aubépine, trèfle, saule, cornouiller sanguin... La quantité de pollen de colza quantifiée est supérieure à $70 \%$ pour seulement 1 prélèvement sur 17.

Autre observation intéressante : un des ruchers est divisé en deux parties séparées par une centaine de mètres. Ces deux groupes présentent des sources $d^{\prime}$ alimentation différentes : colza et aubépine pour I'un et trèfle pour l'autre (figures 3 et 4).

\section{Conclusion et perspectives}

Ce suivi a révélé une interruption de ponte pour 30 et $40 \%$ des colonies de 2 ruchers ce qui a conduit à leur effondrement plus tard dans la saison. Ces problèmes de mortalités ont été observés par d'autres apiculteurs de la région Centre et ont fait l'objet d'une enquête nationale par la Brigade vétérinaire. Un troisième rucher a souffert de problèmes de gestion apicole quand au quatrième, $50 \%$ des populations sont populeuses, les autres dans un état assez variable.

Les analyses des pratiques agricoles, des pollens, des abeilles et l'observation complète des colonies n'ont pas permis d'identifier des causes d'affaiblissement ou de mortalité. Ce suivi a cependant permis de connaître les pratiques sanitaires apicoles et agricoles autour des ruchers et d'associer les expertises des professionnels agricoles et apicoles. II en ressort que la réglementation concernant les pratiques agricoles est globalement bien respectée.

Cependant, ce dispositif ne permet pas $d^{\prime}$ 'étudier les interactions entre facteurs ainsi que l'impact d'autres facteurs non connus qui pourraient peut-être expliquer les phénomènes observés. Par exemple, les conditions météorologiques peuvent être un élément explicatif. Néanmoins, même si les mortalités sont survenues après un brusque refroidissement, les données récoltées ne permettent pas de conclure quand à l'impact des conditions météorologiques sur les populations.

Ce suivi a mis en évidence la nécessité d'une étude plus globale à la fois dans le 


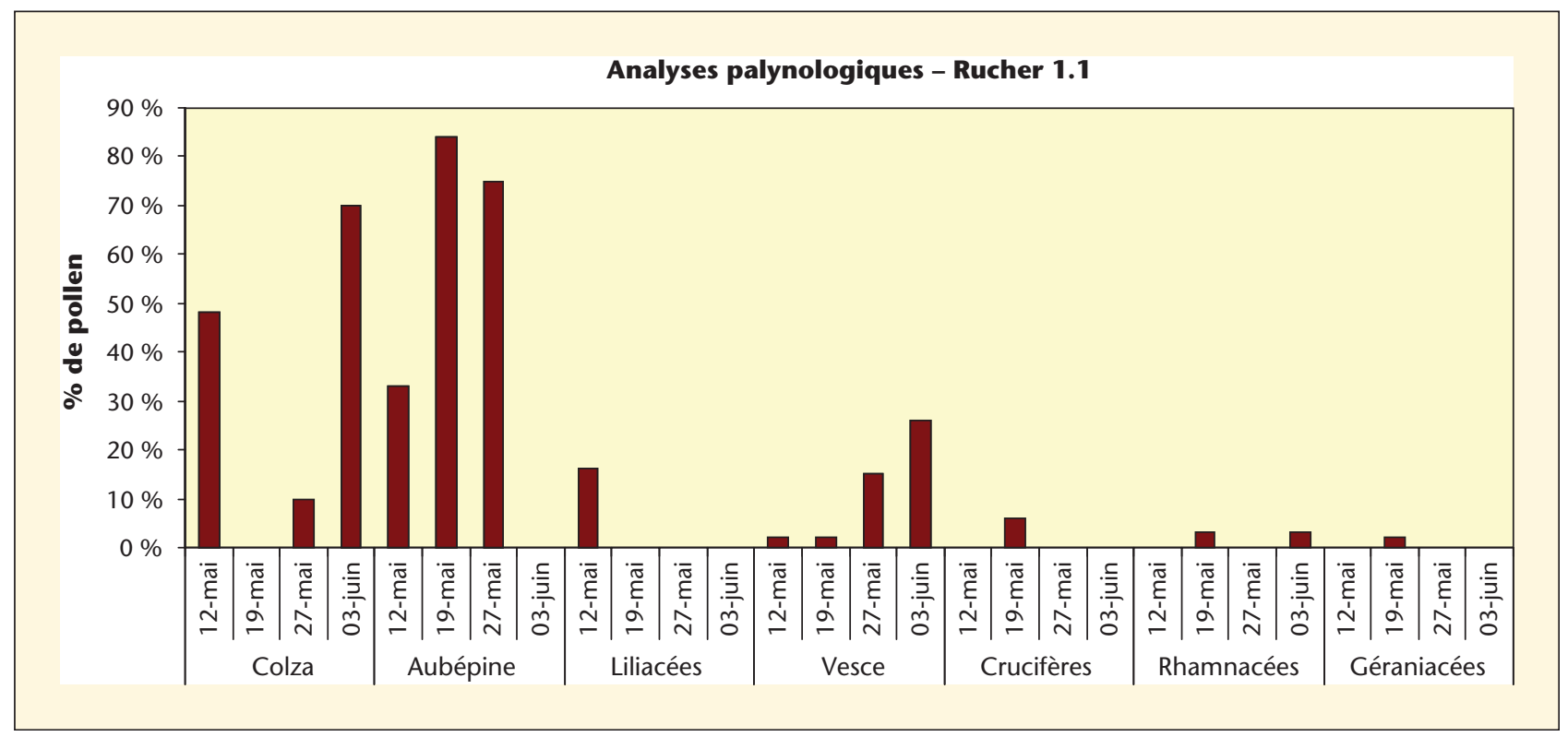

Figure 3. Composition du pollen de la partie 1 du rucher 1 à différentes dates au cours de la floraison du colza.

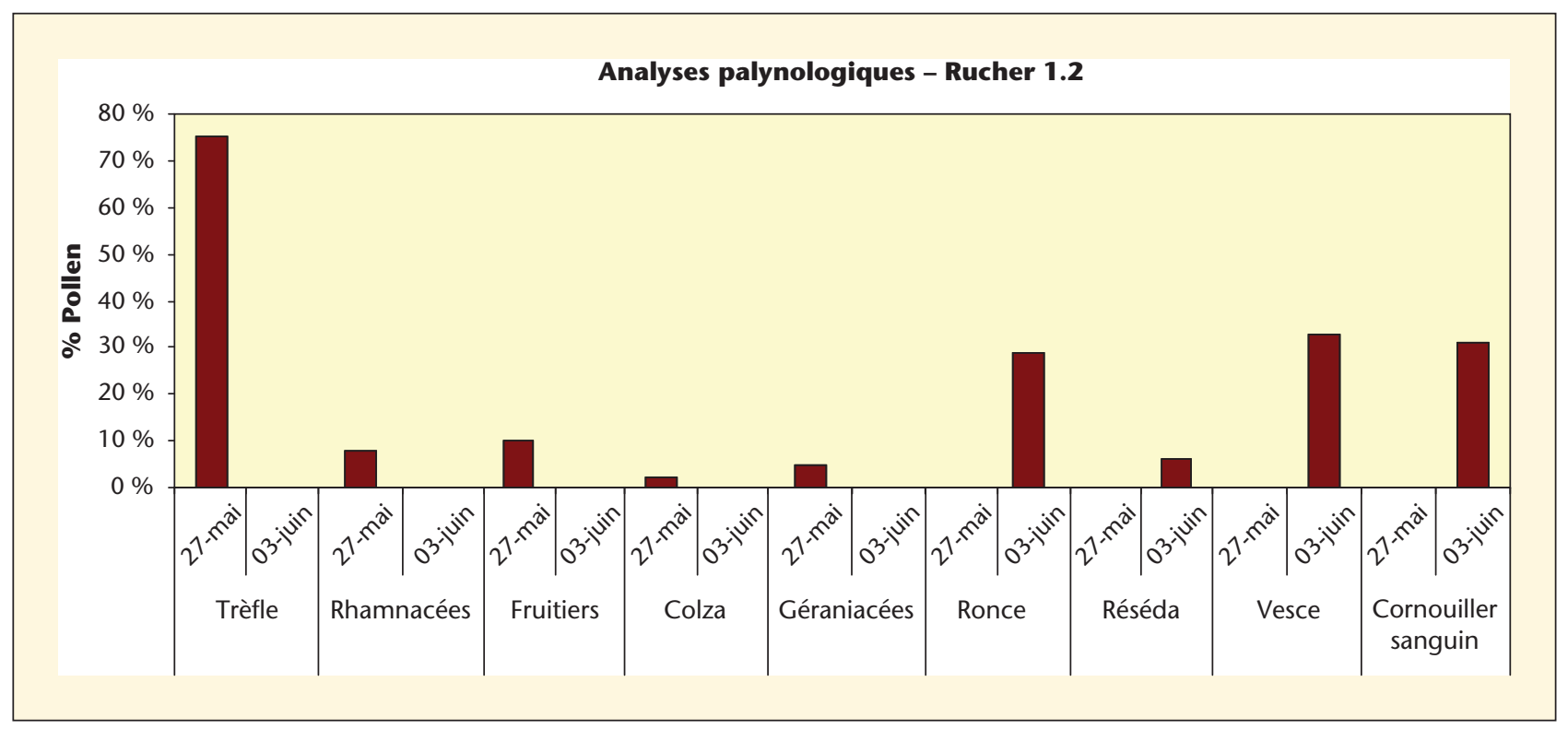

Figure 4. Composition du pollen de la partie 2 du rucher 1 à différentes dates au cours de la floraison du colza.

temps et dans l'espace, qui dans l'idéal intégrerait les historiques des ruches sur le long terme ainsi qu'une description précise du paysage et des pratiques sur l'ensemble des cultures.

La création d'un réseau national de ruchers sur le long terme, inexistant à I'heure actuelle, serait le moyen de dresser un état des lieux sur les problèmes de mortalités anormales des colonies.
Cela nécessiterait la mise en commun des compétences de manière à mettre en place une méthodologie appropriée et approuvée par tous, manquante à ce jour.

\section{RÉFÉRENCES}

Klein $\mathrm{AM}$, Vaissière $\mathrm{BE}$, Cane $\mathrm{JH}$, et al. Importance of pollinators in changing landscapes for world crops. Proc $R$ Soc B 2007 ; $274: 303-13$.
Mollier P, Sarazin M, Savini I. Le déclin des abeilles, un casse-tête pour la recherche. INRA Magazine 2009 ; 9 : 13-24.

Pierre J, Renard M. Bilan de 30 ans de travaux de recherche effectués en France sur la pollinisation du colza. OCL $2010 ; 17$ : 121-6.

GEM-ONIFLHOR. Audit de la filière miel 2005. 2005. 